\title{
Inactivation of the Huntington's disease gene (Hdh) impairs anterior streak formation and early patterning of the mouse embryo
}

\author{
Juliana M Woda ${ }^{1}$, Teresa Calzonetti ${ }^{1}$, Paige Hilditch-Maguire ${ }^{3}$, \\ Mabel P Duyao ${ }^{4}$, Ronald A Conlon ${ }^{2}$ and Marcy E MacDonald ${ }^{* 1}$
}

Address: ${ }^{1}$ Molecular Neurogenetics Unit, Center for Human Genetic Research, Massachusetts General Hospital, CNY-149, 13th Street, Charlestown MA 02129, USA, 2Department of Genetics, Case Western Reserve University, 10900 Euclid Avenue, Cleveland, OH 44106, USA, ${ }^{3}$ University of Queensland, Faculty of Health Sciences, St Lucia QLD 4072, Australia and ${ }^{4}$ Department of Pathology, Harvard Medical School, 77 Avenue Louis Pasteur, NRB-850A, Boston MA 02115, USA

Email: Juliana M Woda - jwoda@partners.org; Teresa Calzonetti - tcalzon@verizon.net; Paige Hilditch-Maguire - p.hilditchmaguire@uniquest.com.au; Mabel P Duyao - mabel_duyao@hms.harvard.edu; Ronald A Conlon - rac14@po.cwru.edu; Marcy E MacDonald* - macdonam@helix.mgh.harvard.edu

* Corresponding author

Published: 18 August 2005

BMC Developmental Biology 2005, 5:17 doi:10.1186/147/-2/3X-5-17
Received: 21 May 2005

Accepted: 18 August 2005

This article is available from: http://www.biomedcentral.com/I47I-2I3X/5/I7

(c) 2005 Woda et al; licensee BioMed Central Ltd.

This is an Open Access article distributed under the terms of the Creative Commons Attribution License (http://creativecommons.org/licenses/by/2.0), which permits unrestricted use, distribution, and reproduction in any medium, provided the original work is properly cited.

\begin{abstract}
Background: Huntingtin, the HD gene encoded protein mutated by polyglutamine expansion in Huntington's disease, is required in extraembryonic tissues for proper gastrulation, implicating its activities in nutrition or patterning of the developing embryo. To test these possibilities, we have used whole mount in situ hybridization to examine embryonic patterning and morphogenesis in homozygous $H d h h^{e x 4 / 5}$ huntingtin deficient embryos.
\end{abstract}

Results: In the absence of huntingtin, expression of nutritive genes appears normal but E7.0-7.5 embryos exhibit a unique combination of patterning defects. Notable are a shortened primitive streak, absence of a proper node and diminished production of anterior streak derivatives. Reduced Wnt3a, Tbx6 and DIII expression signify decreased paraxial mesoderm and reduced Otx2 expression and lack of headfolds denote a failure of head development. In addition, genes initially broadly expressed are not properly restricted to the posterior, as evidenced by the ectopic expression of Nodal, Fgf8 and Gsc in the epiblast and T (Brachyury) and EvxI in proximal mesoderm derivatives. Despite impaired posterior restriction and anterior streak deficits, overall anterior/ posterior polarity is established. A single primitive streak forms and marker expression shows that the anterior epiblast and anterior visceral endoderm (AVE) are specified.

Conclusion: Huntingtin is essential in the early patterning of the embryo for formation of the anterior region of the primitive streak, and for down-regulation of a subset of dynamic growth and transcription factor genes. These findings provide fundamental starting points for identifying the novel cellular and molecular activities of huntingtin in the extraembryonic tissues that govern normal anterior streak development. This knowledge may prove to be important for understanding the mechanism by which the dominant polyglutamine expansion in huntingtin determines the loss of neurons in Huntington's disease. 


\section{Background}

Huntington's disease (HD) is a dominantly inherited neurodegenerative disorder that is caused by CAG repeats in the $H D$ locus that extend a polyglutamine tract in a ubiquitous HEAT domain protein called huntingtin [1]. The molecular mechanism by which the new property that is conferred on huntingtin by the polyglutamine expansion leads to the hallmark loss of striatal neurons in HD is not known. However, polyglutamine expansions in unrelated proteins that target distinct neuronal cell populations cause distinct 'polyglutamine' neurodegenerative disorders. This observation strongly suggests that the striatal cell specificity of the polyglutamine expansion in the context of huntingtin must be determined by some aspect of huntingtin's structure, subcellular location or activities [2].

Huntingtin is postulated to function as a flexible 350 $\mathrm{kDa}$ HEAT domain scaffold that may facilitate the assembly and possibly the subcellular location of large protein complexes [3-7]. Huntingtin's large number of diverse cytoplasmic and nuclear protein binding partners strongly suggest that huntingtin may participate in a variety of cellular processes that range from trafficking of growth factor complexes to gene transcription (reviewed in $[5,8,9]$. However, despite the potential importance of huntingtin's normal function to our understanding of how the dominant polyglutamine mutation causes HD pathology, huntingtin's precise molecular and cellular activities have not been defined.

Therefore, we, and others, set out to discover huntingtin's essential activities by studying the effects of huntingtin deficiency in the mouse. Inactivation of the mouse HD gene $(H d h)$ has shown that huntingtin is not required for cell viability, as evidenced by the survival of mouse embryonic stem cells and neurons that lack huntingtin [10-12]. However, huntingtin is needed at the level of the organism for proper mammalian embryonic development $[10,13,14]$. Complete lack of huntingtin results in developmental arrest during gastrulation, while severe reduction of huntingtin levels results in abnormal neurogenesis and perinatal lethality [15].

Analysis of huntingtin deficient $H d h^{e x 4 / 5} / H d h^{e x 4 / 5}$ embryos reveals that homozygous inactivation of the mouse $H D$ gene does not overtly affect development until E7.0. By E7.5, mutant embryos exhibit a shortened primitive streak, reduced size and, by morphology, lack a node and head folds. Mutants are rapidly resorbed by E8.0 [10]. Importantly, the expression of huntingtin only in extraembryonic tissues in chimeras rescues this gastrulation phenotype, suggesting that huntingtin is required only in cells of the extraembryonic lineage and acts in a cell nonautonomous manner at this stage [16].
Extraembryonic tissues are essential for supplying nutrients and signals that direct anterior/posterior axis formation and patterning in the developing embryo (reviewed in [17]), implicating huntingtin in either or both of these processes. Of these possibilities, the nutritive role has been more extensively investigated. However, huntingtin deficient embryos do not display obvious visceral endoderm defects, with the notable exception of compromised iron transport in later stage mutants, although iron uptake is undisturbed [16] and endocytosis is not impaired in huntingtin deficient embryos or embryonic stem cells $[16,18]$.

By the same token, huntingtin shuttles through the nucleus, where it is required for proper nuclear localization of its transcription factor partners, suggesting that huntingtin may play a role in transcription cascades in extraembryonic tissues that pattern the embryo [18]. Therefore, we have examined this hypothesis, by monitoring the expression of genes that determine normal embryonic patterning and morphogenesis in $H d h^{e x 4 / 5} / H d h^{e x 4 / 5}$ huntingtin deficient embryos. Our results support and refine the hypothesis, indicating that huntingtin is required for proper mesoderm patterning and for normal regional restriction of the expression of a subset of growth and transcription factors.

\section{Results \\ Huntingtin-deficient embryos exhibit abnormal streak progression and paraxial mesoderm production}

Since extraembryonic tissues supply nutrients to the developing embryo, we tested the possibility that huntingtin deficiency may perturb this function by performing RT-PCR analysis to examine the expression of a panel of 'nutritive' genes in E7.5 wild-type and Hdhex4/5/Hdhex4/5 huntingtin deficient embryos. Consistent with a previous report [16], no obvious differences were found in the expression of "nutritive" genes (Hnf4, Afp, Tfn, ApoAI, Apo$A I V$, and $A p o B$ ) or genes involved in yolk sac hematopoiesis or vasculogenesis (Ttr, Rbp, Flt1, Flk1, Tal1, Rbtn2, GATA1) (data not shown), suggesting that huntingtin is not essential for the proper expression of genes required for the nutritive function of the extraembryonic tissues.

To investigate huntingtin's developmental activities, we then analyzed the expression of genes which pattern the early embryo or mark morphogenic landmarks in wildtype and $H d h^{e x 4 / 5} / H d h^{e x 4 / 5}$ embryos by whole mount and section in situ hybridization. The dissections confirmed previous morphologic data at E7.0-7.5 that all Hdhex4/5/ $H d h^{e x 4 / 5}$ homozygotes exhibit abnormal morphology, including shortened primitive streak and a lack of morphological head folds or node $[10,13]$. The results of in situ hybridization analysis also confirmed that all three 
germ layers and extraembryonic tissue are formed in huntingtin deficient embryos.

Otx2, normally expressed in the anterior neuroectoderm and anterior visceral endoderm [19], is expressed in mutant embryos at E7.5 (Fig. 1A,B) although the expression domain appears reduced. Similarly, Hesx 1 expression is grossly normal in mutant embryos, with expression localized to the AVE and neuroectoderm (Fig. 1C-F, [20]), although the expression domain also appears reduced. These results indicate appropriate specification and movement of anterior visceral endoderm (AVE) cells from the distal tip and suggest that neuroectoderm is induced in the mutant embryos.

To examine definitive endoderm formation, the expression of $H n f 3 \beta$ (FoxA2) in mutant and wild-type embryos was analyzed. In wild-type embryos, $H n f 3 \beta$ expression is confined to the node and anterior definitive endoderm (Fig. 1G,I[21]). Mutant embryos exhibit $H n f 3 \beta$-reactive definitive endoderm over the disorganized anterior streak region and proceeding rostrally around the distal tip (Fig. $1 \mathrm{H}, \mathrm{J})$. In both normal and mutant embryos, the AVE exhibits little $H n f 3 \beta$ expression. Therefore, huntingtin deficiency does not greatly affect $H n f 3 \beta$ regulation or the reorganization of the visceral endoderm.

The lack of a morphological node and presence of a shortened streak, together with reduced neuroectoderm and lack of headfolds, suggest that anterior streak formation may be impaired in huntingtin deficient embryos. To investigate this possibility, we examined mesoderm formation in mutant embryos. Mesoderm is specified in the mutant embryos, as marked by the expression of $T$ (Brachyury) and Evx1 (Fig. 2A-F). However, close inspection of the data reveals abnormal patterning within this tissue and its derivatives. $T$, normally expressed in the primitive streak, node and axial head process/notochord mesoderm [22], is detected in the shortened streak and axial mesoderm in $H d h^{e x 4 / 5} / H d h^{e x 4 / 5}$ embryos, extending rostrally from a region of weakly positive cells (Fig. 2A,B). T expression appears weaker, however, in the anterior streak, corresponding to cells that will give rise to axial mesoderm (Fig. 2D). $T$ is also ectopically expressed in mutant extraembryonic mesoderm at the anterior embryonic junction and along the chorion (Fig. 2B,D). Similarly, Evx1, normally expressed in primitive streak mesoderm at E7.5 with highest levels in proximal cells [23], is expressed in the proximal shortened streak but is also aberrantly expressed throughout the extraembryonic mesoderm, allantois and chorion (Fig. 2E,F). Extraembryonic mesoderm, derived from the proximal streak, does not normally express $T$ or Evx1 in wild-type embryos [22]. Therefore, the inappropriate expression of $T$ and $E v x 1$, the shortened primitive streak, and the absence of a morpho- logical node, all suggest that the anterior primitive streak is deficient in the mutant embryos.

The anterior streak generates paraxial mesoderm. Therefore we examined paraxial mesoderm formation in wildtype and mutant embryos, revealing deficits in mesoderm patterning. Starting at E.7.5, Wnt3A is expressed in the primitive streak in cells fated to become paraxial mesoderm. In huntingtin deficient mutants, Wnt $3 a$ is induced in the proximal streak (Fig. 2G,H), confirming stage appropriate posterior development, in contrast to the absence of anterior head folds. However, expression of Wnt $3 a$ is noticeably reduced in $H d h^{e x 4 / 5} / H d h^{e x 4 / 5}$ embryos, suggesting a defect in paraxial mesoderm development (Fig. 2H). Reduced expression of Tbx6 in the mesoderm lateral to the primitive streak in mutant embryos confirms this interpretation (Fig. 2I,J). Furthermore, in mutant embryos at E7.5, the expression of Dll1 in the distal streak region and in only a narrow swath of cells located laterally confirms the paucity of paraxial mesoderm (Fig. 2K,L, [24]). These results strongly suggest that anterior primitive streak formation is impaired, resulting in reduced axial and paraxial mesoderm formation and impaired neural development.

\section{Impaired regional restriction of growth factor expression in the absence of huntingtin}

To elucidate the apparent patterning deficits, we next analyzed signaling molecules that are required for early patterning. Nodal, a member of the Tgf $\beta$ family of secreted molecules is required for the formation and maintenance of the primitive streak and induction of the AVE [25-27]. Nodal is normally expressed throughout the epiblast and overlying visceral endoderm at early post implantation stages [28], but later becomes restricted to the posterior of the embryo to the site of primitive streak with asymmetrical visceral endoderm expression marking the left-right axis. By E7.5, Nodal expression is restricted to the node. Nodal expression was assessed in $H d h^{e x 4 / 5} / H d h^{e x 4 / 5}$ embryos heterozygous for the Ndl lac $Z$ allele $[28,29]$. Notably, heterozygous loss of nodal does not alter the $H d h^{e x 4 / 5} / H d h^{e x 4 / 5}$ phenotype, as determined by morphology of $H d h^{e x 4 / 5} / H d h^{e x 4 / 5}: N d l l a c z / N d l+$ embryos compared with $H d h^{e x 4 / 5} / H d h^{e x 4 / 5}$ embryos (data not shown). In contrast to wild-type embryos, which exhibit tight restriction of Nodal.LacZ expression to the node, Hdhex4/5/Hdhex4/ 5:Ndllacz/Ndl+ embryos express Nodal.LacZ throughout the endoderm overlying the epiblast, with higher levels in the posterior in an asymmetric pattern (Fig. 3A-D). The lack of tight restriction of nodal signal is consistent with a failure to form an organized node structure.

F $f 8$ signaling is also essential for normal gastrulation in the mouse embryo. $F g f 8$ is required for cell migration away from the primitive streak [30]. Expressed just prior 

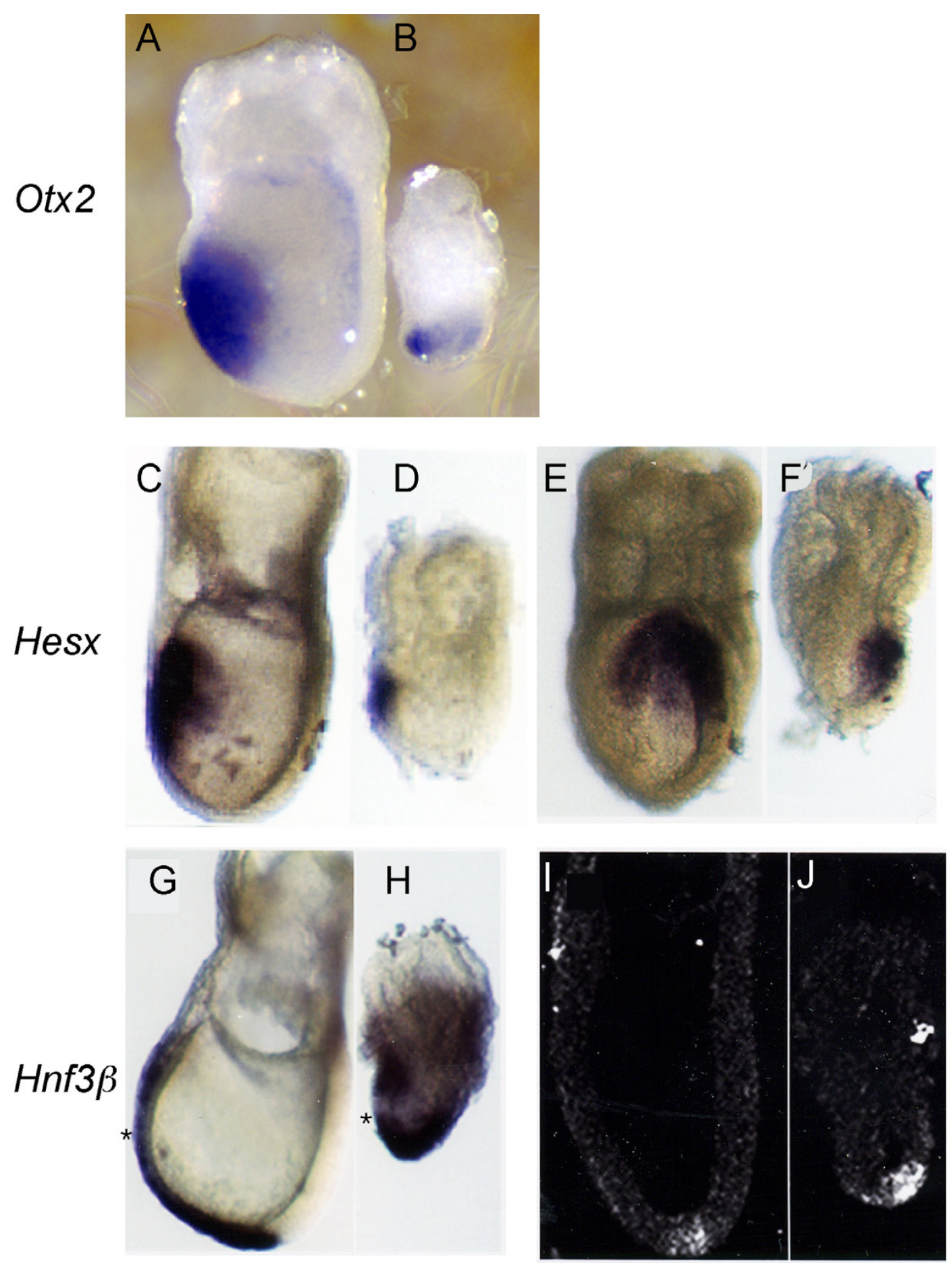

\section{Figure I}

AVE displacement and anterior neurectoderm induction occur normally in the absence of huntingtin. Whole mount in situ hybridization analysis of Otx2 (A,B) and Hesx (C-F) in E7.5 normal $(A, C, E)$ and mutant $(B, D, F)$ embryos reveals that neuroectoderm and anterior visceral endoderm (AVE) develop normally in huntingtin deficient embryos, although the neuroectoderm expression domain is reduced. Asymmetrical expression of Hesx in mutant embryos (F) suggests that left-right transcriptional control is maintained. Hnf3 $\beta$ expression in the definitive endoderm extends around the distal tip and is reduced in the AVE $(*)$ in both normal $(\mathrm{G}, \mathrm{I})$ and mutant embryos $(\mathrm{H}, \mathrm{J})$. Taken together, these results suggest normal ectoderm and endoderm induction and localization in $H d h$ ex4/5/Hdhex4/5 embryos. Embryos are shown in lateral views, with anterior to the left in all pictures with the exception of $E$ and $F$. Embryos are viewed from the anterior aspect in $E$ and $F$. 
$T$
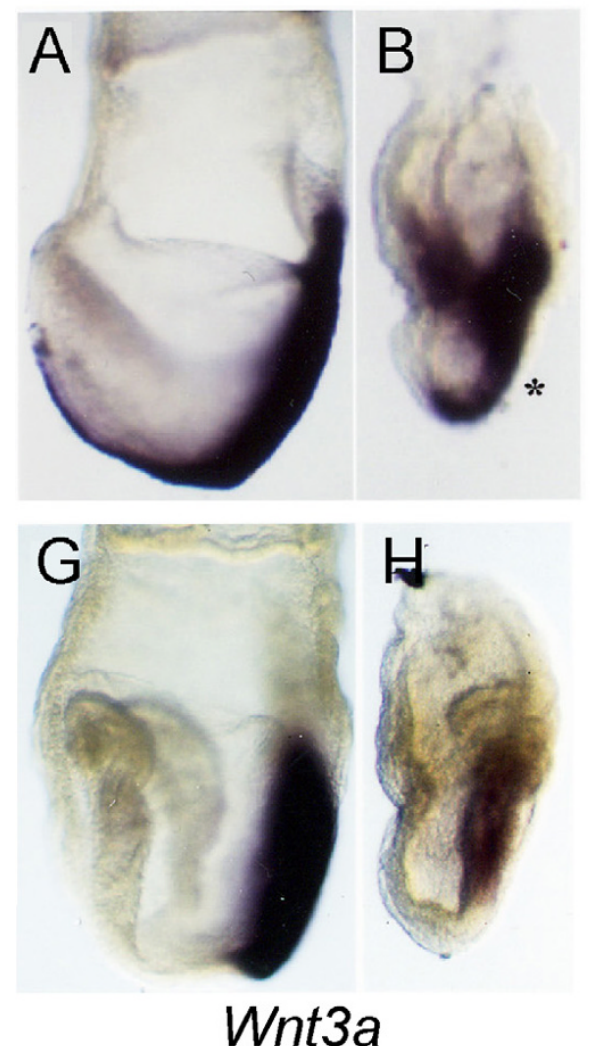
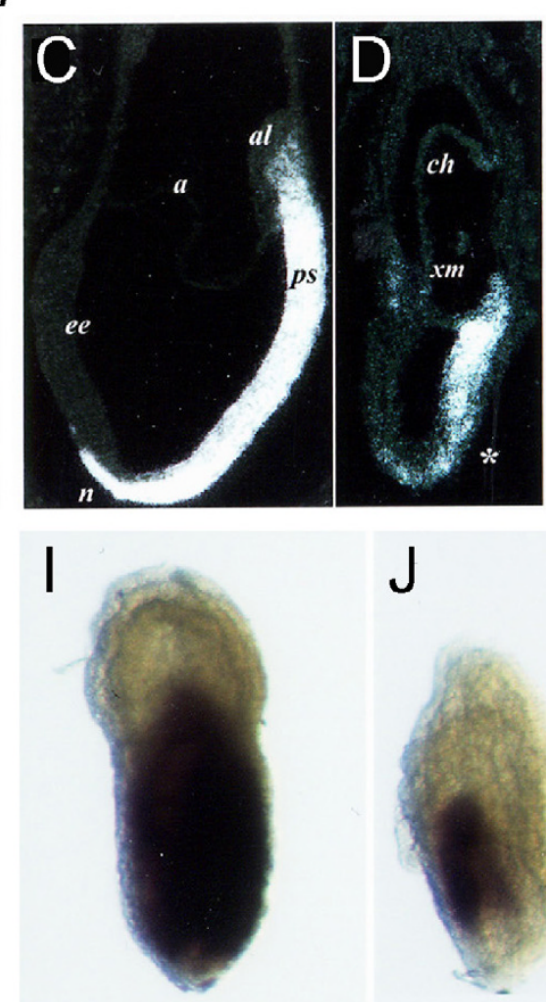

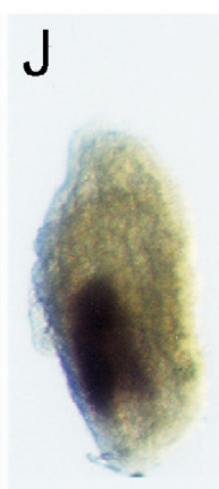

Tbx6
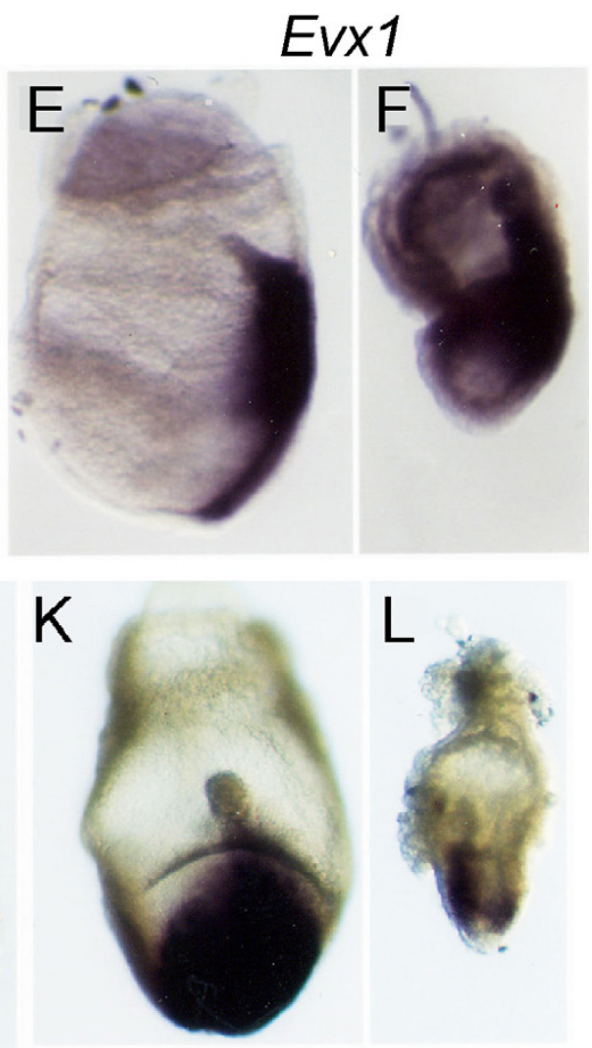

DII1

Figure 2

Huntingtin is required for formation of anterior primitive streak and paraxial mesoderm. Whole mount and section insitu hybridizations of E7.5 embryos shows T (Brachyury) (A-D) is expressed in the primitive streak, node, axial mesoderm and $E v x I(E-F)$ is expressed in the primitive streak, most strongly in the proximal streak wild-type embryos. However, in mutant embryos, both $T(B, D)$ and $E v x I(F)$ are ectopically expressed in the extraembryonic region. Wnt3A expression is reduced in mutant embryos $(\mathrm{H})$, although the localization of its expression to the proximal streak is the same as in wild-type embryos (G). Analysis of paraxial mesoderm markers Tbx6 $(\mathrm{I}, \mathrm{J})$ and $D I I I(\mathrm{~K}, \mathrm{~L})$, reveals that these markers are reduced in mutant embryos (J,L), suggesting impaired paraxial mesoderm production in the absence of huntingtin. Embryos in A-H are shown in a lateral view with anterior oriented to the left. Embryos in $\mathrm{I}-\mathrm{L}$ are shown in a posterior view $(\mathrm{I}, \mathrm{K})$ or near posterior $(\mathrm{J}, \mathrm{L})$ view with proximal oriented toward the top. In (C,D), al = allantois, a = amnion, ch = chorion, ee = embryonic node(N), em $=$ extraembryonic mesoderm, $n=$ node, $\mathrm{ps}=$ primitive streak. Rather than a node, mutant embryos exhibit a region of disorganized cells $(*)$ at the distal extent of the short primitive streak.

to streak formation in the posterior epiblast and visceral endoderm, Fgf8 is restricted to the streak mesoderm at E7.5 in a decreasing proximal-distal gradient and is downregulated in cells shortly after they exit the streak (Fig. $3 \mathrm{E}, \mathrm{G})$. In $H d h^{e x 4 / 5} / H d h^{\text {ex } 4 / 5}$ embryos, Fgf8 expression is strongly expressed in the posterior region in the primitive streak and ectopically in the endoderm overlying the entire epiblast (Fig. 3F,H). However, streak derivatives appear to migrate normally as evidenced by the proper anterior expression of markers such as $\operatorname{Otx} 2, \operatorname{Hnf} 3 \beta$ and Hesx1 anteriorly (Fig. 1). Therefore, mutant embryos exhibit normal migration of streak derivatives but display impaired Fgf8 repression in mutant endoderm.

$H d h^{e x 4 / 5} / H d h^{e x 4 / 5}$ embryos also fail to restrict the expression of goosecoid (Gsc). Normally, Gsc is initially expressed in the visceral endoderm and proximal, posterior streak where the primitive streak will form prior to gastrulation. As the primitive streak forms and extends, Gsc is expressed in the distal streak, the node, and the axial mesoderm extending anteriorly from the node (Fig. 3I,K, [31,32]). However, in the mutant $H d h^{e x 4 / 5} / H d h^{e x 4 / 5}$ embryos, high 

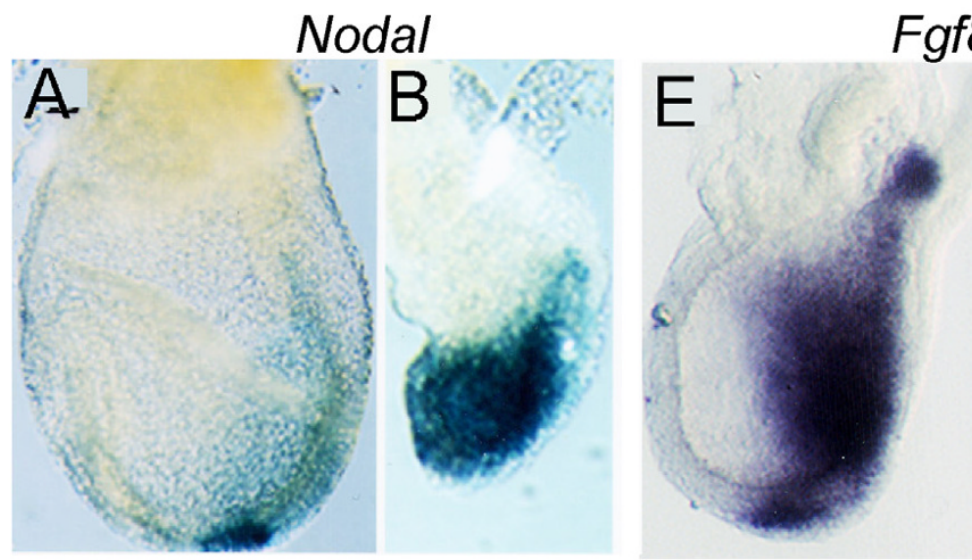

Fgf8
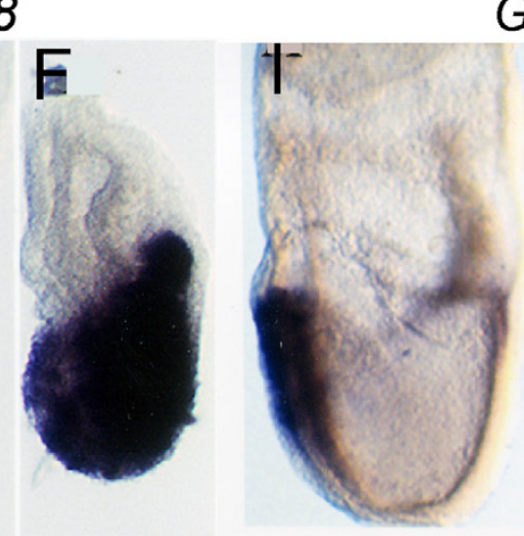

Gsc
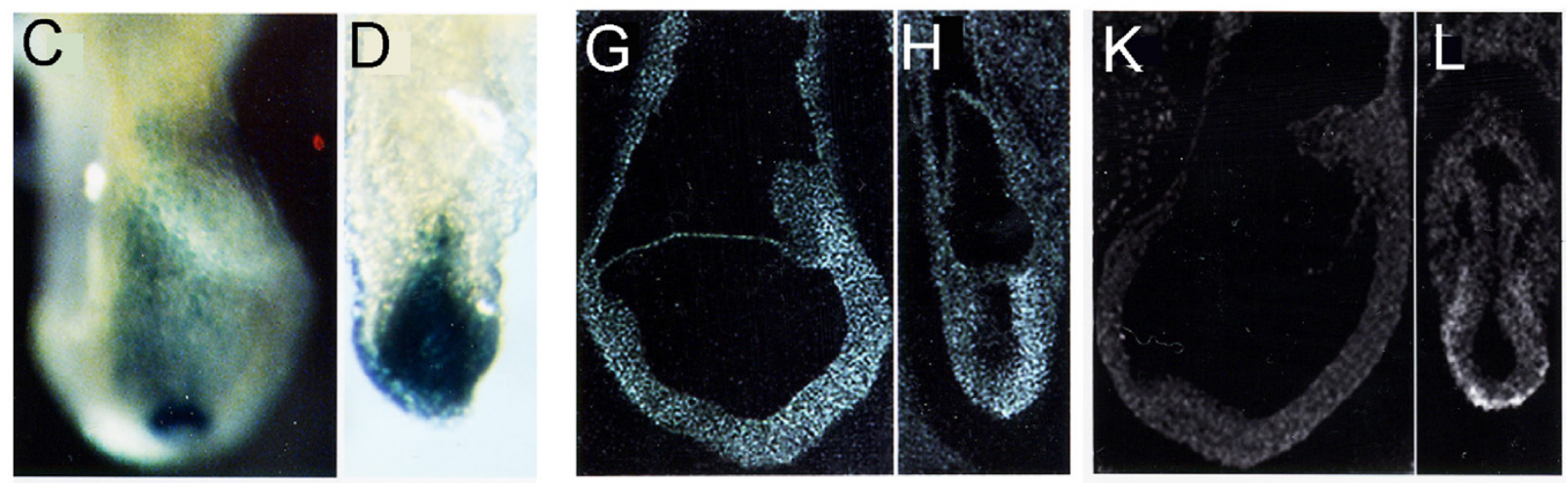

Fgf8

Gsc

\section{Figure 3}

Impaired regional restriction of gene expression in huntingtin deficient embryos. X-gal staining of Nodal-LacZ embryos shows staining in endoderm near the node of normal embryos (,$C)$ but broad staining in mutant embryos (B, $D)$, although expression is higher in the posterior. The tight node expression of Nodal in normal embryos (C) is lost in mutant embryos (D), consistent with the loss of a morphological node in the absence of huntingtin. Whole mount and in situ hybridization of E7.5 day embryos reveals that Fgf8 is detected in the proximal streak and is downregulated in cells migrating out of the streak in normal embryos $(E, G)$. In contrast, Fgf8 remains highly expressed in mutant embryos $(F, H)$. Transient expression of Gsc in the definitive endoderm overlying the prospective head region in normal embryos $(\mathrm{I}, \mathrm{K})$ is distinguished in other cell layers in normal embryos but remains unrestricted in mutant embryos (J,L). Earlier posterior expression of Gsc is also maintained in mutant embryos $(\mathrm{J})$ while it is down-regulated in normal embryos (I). Embryos (A,B,E,F,G,H,I,K,L) are shown in a lateral view with anterior oriented to the left. Embryos (C,D) are in a posterior view.

levels of Gsc expression remain unrestricted in the endoderm overlying the entire embryo and ectopically in cells adjacent to the ectoplacental cone (Fig. 3J,L). These results suggest that, in contrast to proper $H n f 3 \beta$ regulation, Gsc remains inappropriately activated in mutant visceral and definitive endoderm, implicating huntingtin in the proper restriction of this homeodomain transcription factor.

\section{Huntingtin is not required for expression of extraembryonic signaling molecules}

Previous studies of chimeric embryos suggest that huntingtin is required only in the extraembryonic tissue for proper development [16]. Signals from the extraembryonic tissue are critical for the induction of embryonic signals and for patterning the epiblast. Consequently, we examined extraembryonic development in huntingtin deficient embryos. Hnf4 is a transcription factor expressed in the primitive endoderm as soon as this tissue becomes distinct and is a key regulator of visceral endoderm secreted factors such as alphafetoprotein, apolipoproteins, and transferrin. Inactivation of $\mathrm{Hnf4}$ results in impaired gastrulation $[33,34]$. At E7.5, Hnf4 is expressed in the columnar visceral endoderm cells at the extraembryonic-ectoderm junction (Fig. 4A, [33]). In Hdhex4/5/ 

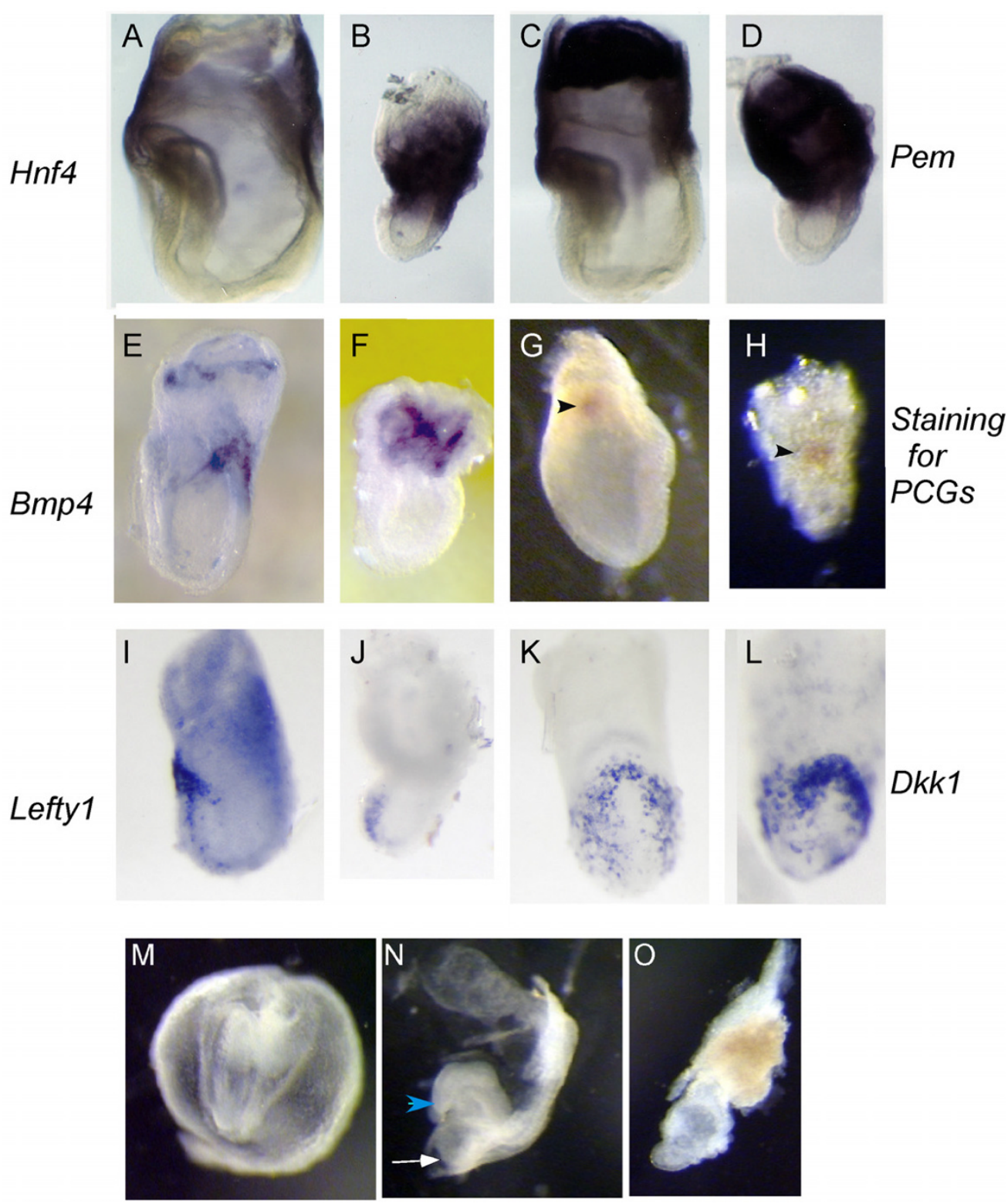

\section{Figure 4}

Normal expression of extraembryonic markers in huntingtin deficient embryos. Whole mount in situ hybridization analysis at E7.5 of markers of the extraembryonic tissues reveals grossly normal expression in the absence of huntingtin. Hnf4, expressed in the visceral endoderm at the junction of embryonic-ectoderm junction $(A)$, is normal in mutant embryos, although the signal is slightly higher (B). Similarly, the expression of Pem transcripts is maintained in mutant embryos (D) similar to normal embryos $(C)$, although Pem is expressed in the abnormal lopsided overhang of visceral endoderm over the anterior of the mutant embryos. Expression of extraembryonic signaling molecules is unaffected by the loss of huntingtin, as evidenced by the expression of Bmp4 (E,F) in the extraembryonic ectoderm, and Leftyl and Dkkl (I-L) in the AVE in mutant embryos. Bmp4 is not localized, however, to a ring of extraembryonic ectoderm in mutant embryos (F) as in normal embryos (E). Primitive germ cells (PCGs) are induced normally in both wild-type $(\mathrm{G})$ and mutant embryos $(\mathrm{H})$, suggesting the Bmp4 signaling from the extraembryonic ectoderm to the epiblast is normal. Leftyl expression appears disorganized in mutant embryos (I) compared to wild-type embryos (J). In contrast, the anterior expression of $D k k l$ in the AVE in mutant embryos (L) matches the wild-type expression pattern (K). Despite normal AVE formation, head folds fail to form in mutant embryos, even when cultured in nutrient rich media for 24 hours. Wild-type E7.5 embryos, when cultured in 75\% rat serum, develop somites (M), heart (white arrow, $\mathrm{N}$ ) and head folds (blue arrow head, $\mathrm{N}$ ) in culture. In contrast, huntingtin deficient embryos continue to live in culture but do not form headfolds, heart or somites (O). Embryos are shown in a lateral view (A-F, I-J) with anterior oriented to the left. Embryos in $(\mathrm{G}, \mathrm{H}, \mathrm{K}, \mathrm{L})$ are shown in an anterior view with proximal oriented up. 
$H d h e^{e x / 5}$ embryos, consistent with normal primitive and visceral endoderm differentiation, Hnf4 expression appears normal, although the signal is stronger in mutant embryos compared to wild-type embryos (Fig. 4B). Similarly, Pem, a transcription factor expressed in proximal visceral endoderm and ectoplacental cone in wild-type embryos at E7.5, also is expressed in these tissues in the mutant embryos (Fig. 4C,D[35]). However, Pem expressing visceral endoderm hangs over the anterior of the mutant embryos, revealing abnormal location despite grossly normal differentiation.

Signals from the extraembryonic tissues, including the anterior visceral endoderm and extraembryonic ectoderm are required for proper formation and patterning of the epiblast [17]. Bmp4 is a signaling molecule that is first expressed uniformly throughout the extraembryonic ectoderm and subsequently is localized to a ring of extraembryonic ectoderm adjacent to the epiblast (Fig. 4E, [36]). A key factor in regulating the formation of the node and primitive streak, Bmp4 is required for patterning the embryo along the proximodistal axis [37-40]. In the absence of huntingtin, Bmp4 expression is properly maintained in the $H d h^{e x 4 / 5} / H d h^{e x 4 / 5}$ extraembryonic ectoderm but is also expressed throughout the extraembryonic ectoderm (Fig. 4F) in a pattern that is similar to early Bmp4 expression rather than being restricted to a ring of extraembryonic ectoderm as seen in the wild-type embryos To assess Bmp4 signaling from the extraembryonic ectoderm, we evaluated primordial germ cells (PGCs), which require Bmp4 for their induction [37]. PGCs can first be detected at E7.0 and subsequently underlie the posterior portion of the primitive streak. Whole mount staining of E7.5 mutant and wild-type embryos for alkaline phosphatase activity reveals that PCGs form in $H d h^{e x 4 / 5} / H d h^{e x 4 / 5}$ embryos, suggesting that Bmp4 signaling is functional in the absence of huntingtin (Fig. 4G,H).

The anterior visceral endoderm (AVE) is also an extraembryonic source of signals that are critical for early patterning. Wnt and nodal antagonists, Dkk1 (mdkk-1) and Lefty 1 respectively, are expressed in the AVE and are important in limiting the posteriorization of the anterior embryo by restricting Nodal and Wnt signaling [41-43]. In $H d h^{e x 4 / 5} / H d h^{e x 4 / 5}$ embryos, both Dkk1 (Fig. 4I,J) and Lefty 1 (Fig. $4 \mathrm{~K}, \mathrm{~L}$ ) are expressed normally in the AVE as compared with wild-type embryos. However, Dkk-1 levels appear to be slightly increased in $H d h^{e x 4 / 5} / H d h^{e x 4 / 5}$ embryos, although the pattern of Dkk-1 expression remains unchanged and this increase may just reflect the same amount of expression in a smaller area. Therefore, the ectopic expression of Nodal (Fig. 3A-D) and the decreased Wnt3a expression (Fig. $2 \mathrm{H}$ ) in mutant embryos do not appear to be result of changes in the expression pattern of Lefty1 or Dkk1.
Despite normal AVE formation and neuroectoderm induction, head folds do not form in Hdhex4/5/Hdhex4/5 embryos. Therefore, to determine whether mutant embryos are inherently capable of forming head folds, embryos harvested at stage E7.5 were allowed to progress in rich culture medium in vitro for 24 hours. Wild-type embryos continued to develop head folds, somites and hearts (Fig. 4M,N). In contrast, mutant stage 7.5 embryos did not develop headfolds, hearts or somites, although these embryos continued to live (Fig. 4O). These results strongly suggest that in the absence of huntingtin, embryos are unable to undergo organogenesis, even if they continue to live past E7.5 in a nutrient rich environment.

\section{Discussion}

We have investigated the embryonic processes that require huntingtin in order to more precisely delineate huntingtin's essential molecular and cellular activities and to provide clues to the mechanism by which the dominant polyglutamine expansion mutation in huntingtin leads to HD pathogenesis. In pursuing the finding that huntingtin is needed only in extraembryonic tissues for normal gastrulation, our data fail to provide evidence of abnormal nutritive gene expression in $H d h^{e x 4 / 5} / H d h^{e x 4 / 5}$ embryos. Instead, our results reveal that huntingtin is required for normal anterior streak formation and the consequent production of paraxial mesoderm, with a previously unrecognized role for huntingtin in the proper extinction of transiently and/or dynamically expressed genes.

Indeed, the hallmark of the huntingtin deficient molecular phenotype is the impaired down-regulation of a subset of dynamically expressed genes, after the proper onset of expression. This phenomenon does not reflect a lack of anterior/posterior axis formation, as evidenced by the formation of the AVE anteriorly and the primitive streak posteriorly. Nor can it be simply explained by delayed development, as stage-specific markers, such as Wnt3a and primordial germ cells, which are detectable at E7.0 in wild-type embryos, are induced appropriately. Furthermore, the expression of $T$ and Evx 1 in the extraembryonic mesoderm of mutant embryos is not a feature of wild-type embryos, even at earlier stages. This strongly suggests that in huntingtin deficient embryos, the migration of the distal streak derivatives to the extraembryonic mesoderm occurs normally but that the down-regulation of these genes is impaired. This impairment may also explain the failure of huntingtin deficient embryos to properly restrict the expression of Fgf8, Nodal and Gsc. Thus, huntingtin may play a direct role in the transcriptional regulation, or mRNA stability of these genes or it may act indirectly by intersecting with other pathways that regulate the expression of these genes. 
The requirement for huntingtin in the extraembryonic tissues had prompted us to test whether impaired extraembryonic signals might be responsible for the dysregulation of gene expression within the epiblast that is observed in $H d h^{e x 4 / 5} / H d h^{e x 4 / 5}$ embryos. Extraembryonic development in $H d h^{e x 4 / 5} / H d h^{e x 4 / 5}$ embryos is associated with mildly elevated levels expression of Hnf4 in the primitive endoderm and Pem in the lopsided anterior chorion but the expression of other known signals, such as Bmp4 from the extraembryonic ectoderm, and Dkk1 and Lefty 1 from the $\mathrm{AVE}$, appear to be normal, although the slight increase in Dkk-1 expression in $H d h^{e x 4 / 5} / H d h^{e x 4 / 5}$ embryos suggests that further investigation into Wnt signaling is warranted. Moreover, extraembryonic Bmp4 signaling is not impaired in the absence of huntingtin, as the induction of PCGs in mutant embryos is normal, implying proper transport and secretion of the appropriate extraembryonic signals. However, Nodal, Fgf8 and Gsc are expressed ectopically in the visceral endoderm of $H d h^{e x 4 / 5} / H d h^{e x 4 / 5}$ embryos. Both Nodal and Fgf8, important growth factors required for normal development of the epiblast, are tightly regulated during gastrulation. Therefore, misexpression of either or both of these factors, or of goosecoid, in the visceral endoderm could contribute to the Hdhex4/5/ $H d h^{e x 4 / 5}$ mutant phenotype. In addition, it is possible that other extraembryonic signal(s) that we have not analyzed may also be affected by the lack of huntingtin activity in extraembryonic cells in mutant embryos.

Huntingtin deficient embryos also fail to form headfolds, and to undergo organogenesis, even after culturing in nutrient rich media. The absence of headfold formation in these embryos does not appear to be a result of a failure to induce neurectoderm or a failure to form the AVE, since mutant embryos express markers such as Otx2, Ddk1, Lefty 1 and Hesx 1 . In addition, since node formation is not required for neural induction [44-46], the failure to form a node in huntingtin deficient embryos is also unlikely to explain the lack of headfolds. The apparent reduction of paraxial mesoderm in $H d h^{e x 4 / 5} / H d h^{e x 4 / 5}$ embryos could explain the lack of headfolds since paraxial mesoderm is important for the full development of neuroectoderm, and consequently, headfolds. Alternatively, the inability to manifest headfolds could suggest that huntingtin is required at a very early stage for normal CNS development. This conclusion is consistent with the finding that severely reduced levels of huntingtin, from a hypomorphic Hdh allele, lead to abnormal brains later in embryonic development [15].

The cardinal features of complete $H d h$ inactivation that we observe are similar to the phenotypes that stem from the complete inactivation of the Polycomb group gene (Pc-g) Eed (embryonic ectoderm development). Indeed, complete deficiency for either huntingtin or the eed protein leads to abnormal streak development, lack of headfold formation, ectopic T, Evx1 and Nodal expression and disruption of anterior primitive streak mesoderm production [47]. Interestingly, Eed protein is also required for proper trophoblast development and normal maintenance of imprinted X-inactivation and genomic imprinting [47-49], suggesting that these activities warrant investigation in huntingtin deficient embryos.

Thus, our observations provide unexpected startingpoints in the search for huntingtin's precise molecular activities, which began with the discovery that this HEAT domain protein hosts the dominant polyglutamine property that is the fundamental basis of HD pathogenesis. In HD patients and in accurate genetic replicas, HD CAG knock-in mice, the dominant mutation specifically affects the major population of neurons in the striatum, without impairing huntingtin's essential activities in embryonic development [50-53]. Indeed, homozygous HD patients make no wild-type huntingtin, and, in the mouse, a single mutant $H d h$ allele's worth of mutant huntingtin can fully rescue huntingtin deficiency embryonic phenotypes $[15,51]$. The quest to understand the HD mechanism, therefore, is aimed at delineating the huntingtin activity that may explain the striatal cell specificity of the polyglutamine mutant version of huntingtin. One hypothesis is that huntingtin is normally involved in gene transcription, as proposed for NRSF/REST mediated BDNF expression [54]. Now, our finding that huntingtin can be absolutely necessary for the appropriate regulation of genes with dynamic expression patterns in vivo, provides a compelling reason to elucidate the cellular machinery that is necessary for huntingtin mediated gene regulation.

\section{Conclusion}

Our findings indicate that huntingtin is required for proper patterning of the epiblast during early embryogenesis, for proper anterior streak and node formation, primitive streak progression, paraxial mesoderm and head fold formation, as well as for the proper restriction of transiently expressed growth and transcription factor genes. Knowledge of the molecular basis of these changes in huntingtin deficient embryos should facilitate the identification of the cellular pathways that are dependent on huntingtin activities. These will be important for implicating candidates to be assessed in the extraembryonic signals that determine anterior streak progression in the developing embryo and in delineating the dominant activity of the polyglutamine tract in huntingtin that determines the striatal specificity of HD.

\section{Methods \\ Mice and genotyping}

The $H d h^{e x 4 / 5}$ mice carrying a pGKneo insertion/replacement inactivating mutation in the mouse HD gene homo- 
logue have been described previously [10]. The experiments were conducted in accordance with an IACUC approved protocol, through the MGH Subcommittee on Animal Research. Mutant $H d h^{e x 4 / 5} / H d h^{e x 4 / 5}$ and normal littermates were obtained in timed pregnancies from mating of $H d h e x 4 / 5 / H d h^{+}$heterozygotes, genotyped by PCR assay, as described [10]. The day of plug was taken to be E0.5. Embryos that were morphologically normal were pooled separately from morphologically mutant embryos for analysis. Nodal expression was determined in embryos from matings of $\mathrm{Hdh}^{e x 4 / 5} / \mathrm{Hdh}^{+} ; \mathrm{NdllacZ} / \mathrm{Ndl}^{+}$ compound heterozygotes genotyped by PCR assay as described in [29].

\section{Whole mount and section in situ hybridization and $\beta$-gal staining}

After dissection in PBS, embryos were fixed overnight in $4 \%$ paraformaldehyde at $4{ }^{\circ} \mathrm{C}$. For sections, decidua fixed in $4 \%$ paraformaldehyde, were embedded in paraffin and sectioned at 7 microns. RNA in situ hybridizations were performed as described previously [55]. Nodal.lacZ expression was assessed by $\beta$-galactosidase staining as reported [29], on embryos post fixed in $4 \%$ paraformaldehyde. Embryos were mounted in $80 \%$ glycerol before being photographed.

The huntingtin deficient phenotype is fully penetrant at each of the stages that were assessed [10]. Three to six embryos were evaluated for each marker, with every embryo exhibiting the same mutant phenotype in each case.

\section{Alkaline phosphatase staining of Primordial Germ Cells (PCGs)}

After dissections, embryos were fixed in 4\% paraformaldehyde briefly and washed and stored in $1 \times \mathrm{PBS} / 0.1 \% \mathrm{TX}$ 100 at $4^{\circ} \mathrm{C}$. Embryos were washed once with Tris-Maleate Buffer (25 mM Tris-Maleate, $\mathrm{pH}=9.0,0.8 \mathrm{mM} \mathrm{MgCl}_{2}$ ) and were subsequently incubated in alkaline phosphatase staining solution (25 mM Tris-Maleate, $\mathrm{pH}=9.0,0.8 \mathrm{mM}$ $\mathrm{MgCl}_{2}, 0.4 \mathrm{mg} / \mathrm{ml}$ alpha-naphthyl phosphate, $1 \mathrm{mg} / \mathrm{ml}$ Fast-Red). Stained embryos were washed in $1 \times \mathrm{PBS} / 0.1 \%$ TX-100.

\section{Whole embryo culture}

Embryos were dissected at E7.5 and washed in DMEM. Embryos were then cultured individually in $1 \mathrm{ml}$ of culture media (75\% immediately centrifuged rat serum and $25 \%$ DMEM [56]) for 24 hours while rotating in a $37^{\circ} \mathrm{C}$ incubator in $5 \% \mathrm{CO}_{2}$. Embryos were then fixed in $4 \%$ paraformaldehyde for analysis.

\section{Abbreviations}

AVE, anterior visceral endoderm; HD, Huntington's disease gene; HD, Huntington's disease; Hdh, mouse HD gene homologue; PCGs, primordial germ cells

\section{Authors' contributions}

JMW, TC, PH-M and MD performed whole mount and in situ hybridization assays. MEM and RC contributed to the conception of this study. JMW, TC, PH-M and MEM drafted the manuscript and RC contributed to its finalization. All authors read and approved the final manuscript.

\section{Acknowledgements}

We are grateful to Drs. A. Gossler, J. Darnell, Jr., J Rossant, G. Keller, S. Orkin, G. Martin, T. Yamaguchi, A. McMahon, R. Maas, K., Muneoka, A. Simeone, Hamada $\mathrm{H}$. and $\mathrm{C}$. Niehrs for the generous gifts of clones and antibody reagents and Dr. E. Robertson for NdllacZ mice. We would like to thank Kathy Molyneaux for her helpful suggestions and technical assistance. We also thank Vladimir Vrbanac, Janice Espinola and Edith Toral Lopez for assistance with animal husbandry. We also thank the members of the MacDonald lab for helpful discussions during the completion of this work. This work was supported by the NINDS grants NS32765 and NSI6367, and grants from the Foundation for the Care and Cure of Huntington's disease and with the support of the Huntington's Disease Society of America Coalition for the Cure and the Hereditary Disease Foundation. Juliana M. Woda is the recipient of the Milton Wexler Postdoctoral Fellowship from the Hereditary Disease Foundation.

\section{References}

I. Huntington's Disease Collaborative Research Group: A novel gene containing a trinucleotide repeat that is expanded and unstable on Huntington's disease chromosomes. Cell 1993, 72:97I-83.

2. Gusella JF, MacDonald ME: Molecular genetics: unmasking polyglutamine triggers in neurodegenerative disease. Nat Rev Neurosci 2000, 1:109-15.

3. Takano H, Gusella JF: The predominantly HEAT-like motif structure of huntingtin and its association and coincident nuclear entry with dorsal, an NF- $\kappa B / R E L /$ dorsal family transcription factor. BMC Neuroscience 2002, 3:15.

4. Andrade MA, Bork P: HEAT repeats in the Huntington's disease protein. Nat Genet 1995, II: I I5-6.

5. MacDonald ME: Huntingtin: alive and well and working in middle management. SCi STKE 2003, 2003:pe48.

6. Marcora E, Gowan K, Lee JE: Stimulation of NeuroD activity by huntingtin and huntingtin-associated proteins HAPI and MLK2. Proc Natl Acad Sci U S A 2003, 100:9578-83.

7. Gauthier LR, Charrin BC, Borrell-Pages M, Dompierre JP, Rangone H, Cordelieres FP, De Mey J, MacDonald ME, Lessmann V, Humbert S, et al: Huntingtin controls neurotrophic support and survival of neurons by enhancing BDNF vesicular transport along microtubules. Cell 2004, I I 8: 127-38.

8. Harjes $\mathrm{P}$, Wanker $\mathrm{EE}$ : The hunt for huntingtin function: interaction partners tell many different stories. Trends Biochem Sci 2003, 28:425-33.

9. Gusella JF, MacDonald ME: Huntingtin: a single bait hooks many species. Current Opinion in Neurobiology 1998, 8:425-30.

10. Duyao MP, Auerbach AB, Ryan A, Persichetti F, Barnes GT, McNeil SM, Ge P, Vonsattel JP, Gusella JF, Joyner AL: Inactivation of the mouse Huntington's disease gene homolog Hdh. Science 1995, 269:407-I0.

II. Metzler M, Helgason CD, Dragatsis I, Zhang T, Gan L, Pineault N, Zeitlin SO, Humphries RK, Hayden MR: Huntingtin is required for normal hematopoiesis. Human Molecular Genetics 2000, 9:387-94.

12. Metzler M, Chen N, Helgason CD, Graham RK, Nichol K, McCutcheon K, Nasir J, Humphries RK, Raymond LA, Hayden MR: Life without huntingtin: normal differentiation into functional neurons. Journal of Neurochemistry 1999, 72:1009-18. 
13. Zeitlin S, Liu JP, Chapman DL, Papaioannou VE, Efstratiadis A Increased apoptosis and early embryonic lethality in mice nullizygous for the Huntington's disease gene homologue. Nature Genetics 1995, I I: I55-63.

14. Nasir J, Floresco SB, O'Kusky JR, Diewert VM, Ricmhan JM, Zeisler J, Borowski A, Marth JD, Phillips AG, Hayden MR: Targeted disruption of the Huntington's disease gene results in embryonic lethality and behavioral and morphological changes in heterozygotes. Cell |995, 81:8||-23.

15. White JK, Auerbach W, Duyao MP, Vonsattel JP, Gusella JF, Joyner $A L$, MacDonald ME: Huntingtin is required for neurogenesis and is not impaired by the Huntington's disease CAG expansion. Nature Genetics 1997, I 7:404-10.

16. Dragatsis I, Efstratiadis A, Zeitlin S: Mouse mutant embryos lacking huntingtin are rescued from lethality by wild-type extraembryonic tissues. Development 1998, I 25: 1529-39.

17. Beddington RS, Robertson EJ: Anterior patterning in mouse. Trends Genet 1998, 14:277-84.

18. Hilditch-Maguire P, Trettel F, Passani LA, Auerbach A, Persichetti F, MacDonald ME: Huntingtin: an iron-regulated protein essential for normal nuclear and perinuclear organelles. Human Molecular Genetics 2000, 9:2789-97.

19. Simeone A, Acampora D, Mallamaci A, Stornaiuolo A, D'Apice MR Nigro V, Boncinelli E: A vertebrate gene related to orthodenticle contains a homeodomain of the bicoid class and demarcates anterior neuroectoderm in the gastrulating mouse embryo. Embo J 1993, I 2:2735-47.

20. Thomas P, Beddington R: Anterior primitive endoderm may be responsible for patterning the anterior neural plate in the mouse embryo. Curr Biol 1996, 6: 1487-96.

21. Ang SL, Wierda A, Wong D, Stevens KA, Cascio S, Rossant J, Zaret KS: The formation and maintenance of the definitive endoderm lineage in the mouse: involvement of HNF3/forkhead proteins. Development 1993, I| 19:130|-I5.

22. Wilkinson DG, Bhatt S, Herrmann BG: Expression pattern of the mouse $\mathbf{T}$ gene and its role in mesoderm formation. Nature 1990, 343:657-9.

23. Dush MK, Martin GR: Analysis of mouse Evx genes: Evx-I displays graded expression in the primitive streak. Dev Biol 1992, | 5 |:273-87.

24. Bettenhausen B, Hrabe de Angelis M, Simon D, Guenet JL, Gossler A: Transient and restricted expression during mouse embryogenesis of DII I, a murine gene closely related to Drosophila Delta. Development 1995, I 2 I:2407-18.

25. Zhou X, Sasaki $H$, Lowe $L$, Hogan BL, Kuehn MR: Nodal is a nove TGF-beta-like gene expressed in the mouse node during gastrulation. Nature 1993, 36 I:543-7.

26. Conlon FL, Lyons KM, Takaesu N, Barth KS, Kispert A, Herrmann B Robertson EJ: A primary requirement for nodal in the formation and maintenance of the primitive streak in the mouse. Development 1994, I 20:1919-28.

27. Schier AF, Shen MM: Nodal signalling in vertebrate development. Nature 2000, 403:385-9.

28. Varlet I, Collignon J, Norris DP, Robertson EJ: Nodal signaling and axis formation in the mouse. Cold Spring Harb Symp Quant Biol 1997, 62:105-13.

29. Collignon J, Varlet I, Robertson EJ: Relationship between asymmetric nodal expression and the direction of embryonic turning. Nature 1996, 38 I: |55-8.

30. Sun X, Meyers EN, Lewandoski M, Martin GR: Targeted disruption of Fgf8 causes failure of cell migration in the gastrulating mouse embryo. Genes Dev 1999, 13:1834-46.

31. Filosa S, Rivera-Perez JA, Gomez AP, Gansmuller A, Sasaki H, Behringer RR, Ang SL: Goosecoid and HNF-3beta genetically interact to regulate neural tube patterning during mouse embryogenesis. Development 1997, 1 24:2843-54.

32. Blum M, Gaunt SJ, Cho KW, Steinbeisser H, Blumberg B, Bittner D, De Robertis EM: Gastrulation in the mouse: the role of the homeobox gene goosecoid. Cell 1992, 69:1097-106.

33. Chen WS, Manova K, Weinstein DC, Duncan SA, Plump AS, Prezioso VR, Bachvarova RF, Darnell JE Jr: Disruption of the HNF-4 gene, expressed in visceral endoderm, leads to cell death in embryonic ectoderm and impaired gastrulation of mouse embryos. Genes Dev 1994, 8:2466-77.

34. Duncan SA, Nagy A, Chan W: Murine gastrulation requires HNF-4 regulated gene expression in the visceral endoderm: tetraploid rescue of Hnf-4(-/-) embryos. Development 1997 I 24:279-87

35. Lin TP, Labosky PA, Grabel LB, Kozak CA, Pitman JL, Kleeman J, MacLeod CL: The Pem homeobox gene is $\mathbf{X}$-linked and exclusively expressed in extraembryonic tissues during early murine development. Dev Biol 1994, 166: 170-9.

36. Waldrip WR, Bikoff EK, Hoodless PA, Wrana JL, Robertson EJ: Smad2 signaling in extraembryonic tissues determines anterior-posterior polarity of the early mouse embryo. Cell I998, 92:797-808

37. Lawson KA, Dunn NR, Roelen BA, Zeinstra LM, Davis AM, Wright $\mathrm{CV}$, Korving JP, Hogan BL: Bmp4 is required for the generation of primordial germ cells in the mouse embryo. [comment]. Genes \& Development 1999, 13:424-36.

38. Fujiwara T, Dehart DB, Sulik KK, Hogan BL: Distinct requirements for extra-embryonic and embryonic bone morphogenetic protein 4 in the formation of the node and primitive streak and coordination of left-right asymmetry in the mouse. Development 2002, I 29:4685-96.

39. Fujiwara T, Dunn NR, Hogan BL: Bone morphogenetic protein 4 in the extraembryonic mesoderm is required for allantois development and the localization and survival of primordial germ cells in the mouse. Proceedings of the National Academy of Sciences of the United States of America 200I, 98:13739-44.

40. Winnier G, Blessing M, Labosky PA, Hogan BL: Bone morphogenetic protein-4 is required for mesoderm formation and patterning in the mouse. Genes \& Development 1995, 9:2105-16.

4I. Sakuma R, Ohnishi Yi Y, Meno C, Fujii H, Juan H, Takeuchi J, Ogura $\mathrm{T}$, Li E, Miyazono K, Hamada H: Inhibition of Nodal signalling by Lefty mediated through interaction with common receptors and efficient diffusion. Genes Cells 2002, 7:40I-I2.

42. Perea-Gomez A, Lawson KA, Rhinn M, Zakin L, Brulet P, Mazan S, Ang SL: Otx2 is required for visceral endoderm movement and for the restriction of posterior signals in the epiblast of the mouse embryo. Development 200I, I 28:753-65.

43. Perea-Gomez A, Rhinn M, Ang SL: Role of the anterior visceral endoderm in restricting posterior signals in the mouse embryo. Int J Dev Biol 200I, 45:3 I I-20.

44. Klingensmith J, Ang SL, Bachiller D, Rossant J: Neural induction and patterning in the mouse in the absence of the node and its derivatives. Dev Biol 1999, 2 1 6:535-49.

45. Episkopou V, Arkell R, Timmons PM, Walsh J], Andrew RL, Swan D: Induction of the mammalian node requires Arkadia function in the extraembryonic lineages. Nature 200I, 4 1 0:825-30.

46. Ang SL, Rossant J: HNF-3 beta is essential for node and notochord formation in mouse development. Cell 1994, 78:56|-74.

47. Faust C, Schumacher A, Holdener B, Magnuson T: The eed mutation disrupts anterior mesoderm production in mice. Development 1995, I 2 1:273-85.

48. Morin-Kensicki EM, Faust C, LaMantia C, Magnuson T: Cell and tissue requirements for the gene eed during mouse gastrulation and organogenesis. Genesis 200 I, 3 I: |42-6.

49. Wang J, Mager J, Chen Y, Schneider E, Cross JC, Nagy A, Magnuson $\mathrm{T}$ : Imprinted $\mathbf{X}$ inactivation maintained by a mouse Polycomb group gene. Nat Genet 200I, 28:37I-5.

50. Folstein SE: Huntington's Disease: A Disorder of Families. Baltimore, MD: Johns Hopkins Press; 1989.

5I. Lin CH, Tallaksen-Greene S, Chien WM, Cearley JA, Jackson WS, Crouse AB, Ren S, Li XJ, Albin RL, Detloff PJ: Neurological abnormalities in a knock-in mouse model of Huntington's disease. Human Molecular Genetics 200I, I 0:137-44.

52. Vonsattel JP, DiFiglia M: Huntington disease. I Neuropathol Exp Neurol 1998, 57:369-84.

53. Wheeler VC, Gutekunst CA, Vrbanac V, Lebel LA, Schilling G, Hersch S, Friedlander RM, Gusella JF, Vonsattel JP, Borchelt DR, et al.: Early phenotypes that presage late-onset neurodegenerative disease allow testing of modifiers in Hdh CAG knock-in mice. Human Molecular Genetics 2002, I I:633-40.

54. Zuccato C, Tartari M, Crotti A, Goffredo D, Valenza M, Conti L, Cataudella $T$, Leavitt BR, Hayden MR, Timmusk T, et al.: Huntingtin interacts with REST/NRSF to modulate the transcription of NRSE-controlled neuronal genes. Nat Genet 2003, 35:76-83.

55. Conlon RA, Herrmann BG: Detection of messenger RNA by in situ hybridization to postimplantation embryo whole mounts. Methods Enzymol 1993, 225:373-83. 
56. Sturm K, Tam PP: Isolation and culture of whole postimplantation embryos and germ layer derivatives. Methods Enzymol 1993, 225:164-90

Publish with Bio Med Central and every scientist can read your work free of charge

"BioMed Central will be the most significant development for disseminating the results of biomedical research in our lifetime. " Sir Paul Nurse, Cancer Research UK

Your research papers will be:

- available free of charge to the entire biomedical community

- peer reviewed and published immediately upon acceptance

- cited in PubMed and archived on PubMed Central

- yours - you keep the copyright

Submit your manuscript here:

http://www.biomedcentral.com/info/publishing_adv.asp
Biomedcentral 